\title{
Do Health Educator Telephone Calls Reduce At-risk Drinking Among Older Adults in Primary Care?
}

\author{
James C. Lin, MD, MBA ${ }^{1,2,3}$, Mitchell P. Karno, $P h D^{4}$, Lingqi Tang, PhD ${ }^{5}$, Kristen L. Barry, PhD \\ Frederic C. Blow, PhD 6 , James W. Davis, $M D^{2}$, Karina D. Ramirez, $B A^{2}$, Sandra Welgreen, $M D^{7}$, \\ Marc Hoffing, $M D^{8}$, and Alison A. Moore, MD, MPH $2,4,9$
}

\begin{abstract}
'Special Fellowship in Advanced Geriatrics, VA Greater Los Angeles Healthcare System, Los Angeles, CA, USA; ${ }^{2}$ Department of Medicine, David Geffen School of Medicine at UCLA, Los Angeles, CA, USA; ${ }^{3}$ Department of Medicine, Cheng Ching Hospital, Taichung, Taiwan; ${ }^{4}$ Integrated Substance Abuse Programs, UCLA, Los Angeles, CA, USA; ${ }^{5}$ Health Services Research Center, Semel Institute for Neuroscience and Human Behavior, UCLA, Los Angeles, CA, USA; ${ }^{6}$ Department of Psychiatry, University of Michigan, Ann Arbor, MI, USA; ${ }^{7}$ Kaiser Permanente, Panorama City, CA, USA; ${ }^{8}$ Desert Oasis Healthcare, Palm Springs, CA, USA; ${ }^{9}$ Division of Geriatric Medicine, Los Angeles, CA, USA.
\end{abstract}

BACKGROUND: Alcohol screening and brief intervention for unhealthy alcohol use has not been consistently delivered in primary care as part of preventive healthcare.

OBJECTIVE: To explore whether telephone-based intervention delivered by a health educator is efficacious in reducing at-risk drinking among older adults in primary care settings.

DESIGN: Secondary analyses of data from a randomized controlled trial.

PARTICIPANTS: Subjects randomized to the intervention arm of the trial $(\mathrm{n}=310)$.

INTERVENTIONS: Personalized risk reports, advice from physicians, booklet about alcohol and aging, and up to three telephone calls from a health educator. All interventions were completed before the three-month follow-up.

MEASUREMENTS: Risk outcomes (at-risk or not atrisk) at 3 and 12 months after enrollment.

MAIN RESULTS: In univariate analyses, compared to those who remained at risk, those who achieved not atrisk outcome at 3 months were more likely to be women, Hispanic or non-white, have lower levels of education, consume less alcohol, drink less frequently, and have lower baseline number of risks. In mixed-effects logistic regression models, completing all three health educator calls increased the odds of achieving not at-risk outcome compared to not completing any calls at 3 months (OR 5.31; 95\% CI 1.92-14.7; $\mathrm{p}=0.001$ ), but not at 12 months (OR 2.01; 95\% CI 0.71-5.67; $\mathrm{p}=0.18$ ).

CONCLUSIONS: Telephone-based intervention delivered by a health educator was moderately efficacious in reducing at-risk drinking at 3 months after enrollment among older adults receiving a multi-faceted intervention in primary care settings; however, the effect was not sustained at 12 months.

Received August 3, 2009

Revised December 1, 2009

Accepted December 2, 2009

Published online January 26, 2010
KEY WORDS: alcohol; telephone intervention; health educator; at-risk drinking.

J Gen Intern Med 25(4):334-9

DOI: $10.1007 / \mathrm{s} 11606-009-1223-2$

(c) Society of General Internal Medicine 2010

\section{INTRODUCTION}

Unhealthy alcohol use contributes to physical and psychological illnesses, and is a costly public health problem. ${ }^{1-6}$ The magnitude of health consequences associated with unhealthy alcohol use in older drinkers is likely to rise as the proportion and number of older adults increase in the population. ${ }^{7-9}$ For older drinkers, the amount of alcohol use alone is often inadequate to define unhealthy alcohol use. ${ }^{10}$ Physiologic changes associated with aging increase blood alcohol levels for a given dose of alcohol, ${ }^{11}$ and even small quantities of alcohol may have negative effects due to age-associated increases in comorbidities and use of medications. ${ }^{10,12-15}$ Using this paradigm of at-risk drinking, researchers have classified approximately $10 \%$ of older adults as at-risk drinkers in a population-based study and such drinking was associated with increased risk for mortality. ${ }^{16}$

Many clinical trials of brief alcohol intervention have demonstrated short-term reductions in alcohol use. ${ }^{17-20} \mathrm{~A}$ Cochrane review found that brief intervention has been effective in primary care settings in lowering alcohol consumption. ${ }^{21}$ Brief intervention has also been found to decrease healthcare utilization and costs, ${ }^{22}$ and was associated with greater patient-perceived quality of primary care. ${ }^{23}$ National practice guidelines recommend routine use of screening and brief intervention for unhealthy alcohol use. ${ }^{24}$ However, alcohol screening has not been consistently delivered as part of preventive healthcare, ${ }^{25}$ and uptake of brief intervention by primary care physicians has been poor due to various barriers, including lack of time and training. ${ }^{25-29}$ Time limitations during ambulatory care visits are particularly relevant for older adults who may have multiple health concerns and sensory impairments that may further impede the ability of physicians to screen for unhealthy alcohol use and deliver advice. One study found that even when physicians were provided with alcohol screening results and recommendations 
for adult patients who screened positive for hazardous drinking, only $56 \%$ provided any counseling during the visit. ${ }^{30}$ Because of difficulties observed in implementing brief intervention in ambulatory care settings, other modalities to provide intervention have been explored. For example, telephone-based interventions have been studied among drinkers recruited from various clinical settings and found to reduce alcohol use in younger adults. ${ }^{31-33}$

To explore whether telephone-based intervention can help to reduce at-risk drinking among older adults, this study examined data from participants in the intervention arm of a trial designed to test the efficacy of an intervention to reduce drinking in older at-risk drinkers that included telephonebased health education. We hypothesized that participants who completed a series of telephone calls from a health educator would have greater odds of achieving not-at-risk outcome compared to those who did not complete any calls.

\section{METHODS}

\section{Healthy Living as You Age (HLAYA) Study}

The HLAYA study was a 12-month randomized clinical trial designed to test whether screening and brief intervention targeted to at-risk older drinkers in primary care settings can reduce alcohol consumption. The Comorbidity Alcohol Risk Evaluation Tool was used to screen adults aged 55 years and older in participating primary care sites. ${ }^{10,16,34}$ Those identified as at-risk drinkers could have any of seven types of risks: 1) amount of alcohol use, 2) binge drinking ( $\geq 4$ drinks per occasion), 3) driving after drinking, 4) someone being concerned about the subject's drinking, interaction between alcohol and 5) medications, 6) symptoms, or 7) comorbidities. Risk scores, number of risks at baseline, ranged from 1-7. Participating physicians were instructed to indicate whether they gave advice on a study form after seeing their patients who were assigned to the intervention group.

The study enrolled 631 patients from primary care sites in three different healthcare organizations, with 310 patients randomized to the intervention group. At baseline, subjects completed questionnaires with items on sociodemographic, health-related, and alcohol consumption characteristics. The control group received a booklet on general health behaviors, including topics on nutrition, exercise, medication use, alcohol use, and smoking. The intervention subjects received 1) a booklet about alcohol and aging, 2) a personalized feedback report about risks associated with alcohol use, 3) advice from physicians to reduce risks, and 4) up to three telephone calls from a health educator. Risk scores were measured at 3 and 12 months after baseline.

\section{Health Educator Telephone Calls}

All telephone calls were made by one health educator, who had experience working as a community health promoter. She received training from a psychologist with experience in interventions for substance use problems in older adults, and a geriatrician with expertise in alcohol research. The intervention group participants were scheduled to receive three telephone calls at approximately 2, 4, and 8 weeks after baseline visit. During the first call, the health educator reviewed and discussed risks associated with drinking, and used principles of motivational interview to facilitate behavioral change. ${ }^{35}$ The content of the booklet given to subjects at baseline was discussed, providing opportunities to learn more about specific risks. Subjects' drinking patterns, reasons for alcohol consumption, and details regarding any previous attempts to quit were assessed. At the end of the call, the health educator asked about subjects' intentions to reduce alcohol use, and encouraged them to develop a drinking agreement. If a drinking agreement was completed during the first call, it was reviewed during the second and third telephone calls. If no drinking agreement was completed, during the remaining calls, the health educator reiterated the benefits of reducing alcohol use and discussed potential problems associated with initiating behavioral changes. All calls were completed prior to the three-month follow-up. The average length of the first call was approximately 40 minutes; the average lengths of subsequent calls were approximately 20 minutes.

\section{Sociodemographic and Health-related Variables}

Sociodemographic variables examined included age, gender, race (non-Hispanic white or other), living situation (with someone or alone), education ( $\leq$ high school or $>$ high school), income $(\leq \$ 50,000$ or $>\$ 50,000$ annually), and occupation (retired or not retired). Health-related variables included selfrated health status (poor/fair or good/very good/excellent), and ability to do both strenuous and heavy work (yes or no).

\section{Alcohol-related Variables}

Alcohol-related characteristics examined included number of alcoholic drinks consumed per week, frequency of alcohol use (daily or non-daily), baseline risk score (range 1-7), and types of alcohol-related risks. Data from 3- and 12-month follow-up surveys were used to assess number of alcohol-related risks, which was summed as risk scores. Individuals with risk score of 0 were categorized as not at-risk, and individuals with risk scores greater or equal to one were categorized as at-risk.

\section{Intervention-related Variables}

Intervention-related variables assessed included physician documentation of whether advice was provided to reduce or abstain from drinking during the baseline visit (yes or no), and the number of health educator calls the subjects completed (range 0-3). A dichotomous variable indicating whether a subject completed at least one call was constructed (no calls versus at least one call). The number of calls completed were also recoded into three categories (completed no calls, one or two calls, or 3 calls). Completing one or two calls were combined as one category because preliminary analyses found no differences in the main outcome between those who completed one call and those who completed two calls. Completion of a drinking agreement during health educator calls was not introduced as a variable because too few individuals completed a drinking agreement during either the first $(n=10)$ or second $(n=13)$ telephone calls. Dichotomous variables indicating whether an individual completed follow-up surveys at 3 and 12 months were constructed (yes or no). 


\section{Statistical Analyses}

Sociodemographic, health-related, and alcohol consumption characteristics for the entire sample were described using means and standard deviations for continuous variables, and numbers of respondents and percentages for categorical variables. Univariate analyses were used to compare sociodemographic, health-related, and alcohol-related characteristics between those who completed at least one call and those who did not complete any calls. Frequencies and percentages were used to describe risk outcomes for subjects at 3 and 12 months. Univariate analyses were utilized to compare whether there were differences in sociodemographic, healthrelated, alcohol-related, and intervention-related variables between individuals who achieved not at-risk outcome and those who remained at-risk at 3 and 12 months. The chisquare test was used to test for differences among categorical variables, and t-test used for continuous variables. Univariate analyses were also utilized to compare those who completed and those who did not complete the 3- or 12-month surveys.

Multiple logistic regressions with stepwise selection procedures were performed to select variables associated with risk outcomes at 3 and 12 months. Due to differences in completion rates of follow-up surveys between those who did not complete any calls and those who completed at least one call, multivariate analyses were also performed to identify variables associated with completion of follow-up surveys. Age, gender, education, ability to perform strenuous physical activities, baseline risk scores, and physician advice were found to be associated with completion of follow-up surveys (all with $\mathrm{p}<0.2$ ). These variables, along with variables selected using the stepwise procedures, were included in the final models.

To study the association of completing health educator calls with achieving not at-risk outcome at 3 and 12 months, mixedeffects logistic regression model using SAS GLIMMIX procedure
(SAS Institute Inc, Cary, NC) was fit, using follow-up data at 3 and 12 months. Autoregressive AR(1) covariance structure was chosen to account for the within-subject correlation over time. ${ }^{36}$ Completing one or two calls and completing all three calls were compared to completing no calls. Income was not included in the final model due to substantial numbers of missing data for income ( $n=82,26.5 \%$ of sample). Univariate analyses did not find significant differences in income between individuals who achieved not at-risk outcome and those who remained at-risk at 3 months $(p=0.14)$ and 12 months $(p=0.63)$.

\section{RESULTS}

\section{Characteristics of the Sample}

The average age of the sample was 68.7 years (SD 6.8), $71.6 \%$ were men, $88 \%$ were non-Hispanic white, $76.7 \%$ had completed more than a high school education, $73.8 \%$ were living with someone else, and $90 \%$ rated their current health status as good/very good/excellent. At baseline, $67.7 \%$ of these older drinkers drank daily, and the average number of alcoholic beverage consumed per week was 15.2 (SD 7.2). The average number of risks at baseline was 2.9 (SD 1.7). The most common types of alcohol-associated risks were interactions between alcohol and selected a) medications $(71.3 \%$ of the sample), b) symptoms (59.4\%), and c) comorbidities (49.7\%).

Of the total intervention group, $61(19.7 \%$ of the intervention group) did not complete any calls, 93 (30\%) completed one or two calls, and 156 (50.3\%) completed all three calls. The most common reasons for not completing calls were refusal to speak with the health educator $(41.3 \%)$ or inability to reach the individual after three attempts (39.4\%). Univariate analyses comparing sociodemographic, health-related, alcohol consump-

Table 1. Sociodemographic and Health-related Characteristics by Risk Outcomes at 3 and 12 Months

\begin{tabular}{|c|c|c|c|c|c|c|}
\hline & \multicolumn{3}{|l|}{ At 3 months } & \multicolumn{3}{|l|}{ At 12 months } \\
\hline & $\begin{array}{l}\text { Individuals who } \\
\text { are not at risk } \\
(n=125)\end{array}$ & $\begin{array}{l}\text { Individuals } \\
\text { who remained } \\
\text { at risk }(n=122)\end{array}$ & $p$-value & $\begin{array}{l}\text { Individuals who } \\
\text { are not at risk } \\
(n=102)\end{array}$ & $\begin{array}{l}\text { Individuals who } \\
\text { remained at risk } \\
(n=120)\end{array}$ & $p$-value \\
\hline Age, mean \pm SD & $69.2 \pm 6.9$ & $67.8 \pm 6.6$ & 0.12 & $69.3 \pm 6.8$ & $68.3 \pm 6.2$ & 0.22 \\
\hline Sex, n (\%) Men & $86(68.8)$ & $98(80.3)$ & 0.04 & $71(69.6)$ & 87 (72.5) & 0.64 \\
\hline \multicolumn{7}{|l|}{ Race, n (\%) } \\
\hline Non-Hispanic white & $102(81.6)$ & $113(92.6)$ & 0.01 & $88(86.3)$ & 106 (88.3) & 0.65 \\
\hline Hispanic/Non-white & $23(18.4)$ & $9(7.4)$ & & $14(13.7)$ & $14(11.1)$ & \\
\hline \multicolumn{7}{|l|}{ Living situation, n (\%) } \\
\hline With someone & 97 (77.6) & 86 (70.5) & 0.20 & $78(76.5)$ & $87(72.5)$ & 0.50 \\
\hline Alone & $28(22.4)$ & $36(29.5)$ & & $24(23.5)$ & $33(27.5)$ & \\
\hline \multicolumn{7}{|l|}{ Education, n (\%) } \\
\hline Up to high school & $92(73.6)$ & $103(84.4)$ & 0.04 & $28(27.5)$ & $23(19.2)$ & 0.14 \\
\hline More than HS & $33(26.3)$ & $19(15.6)$ & & $74(72.5)$ & $97(80.8)$ & \\
\hline \multicolumn{7}{|l|}{ Income, n (\%) } \\
\hline$\leq \$ 50,000$ & $53(60.9)$ & 47 (50\%) & 0.14 & $44(57.1)$ & $47(53.4)$ & 0.63 \\
\hline \multirow[t]{2}{*}{$>\$ 50,000$} & $34(39.1)$ & 47 (50\%) & & 33 (42.9) & $41(46.6)$ & \\
\hline & (38 missing) & (28 missing) & & (25 missing) & (32 missing) & \\
\hline Occupation, n (\%) & & & & & & 0.59 \\
\hline Non-retired & $30(24)$ & $38(31.2)$ & 0.21 & $27(26.5)$ & $28(23.3)$ & \\
\hline Retired/Homemaker & $95(76)$ & $84(68.8)$ & & 75 (73.5) & $92(76.7)$ & \\
\hline \multicolumn{7}{|l|}{ Self-rated health status, n (\%) } \\
\hline Poor/Fair & $14(11.2)$ & $8(6.6)$ & 0.20 & $12(11.8)$ & $8(6.7)$ & 0.19 \\
\hline Good/Very Good/ Excellent & $111(88.8)$ & $114(93.4)$ & & $90(88.2)$ & $112(93.3)$ & \\
\hline $\begin{array}{l}\text { Able to do both strenuous } \\
\text { and heavy work, n (\%) }\end{array}$ & $93(74.4)$ & $89(73)$ & 0.80 & $80(78.4)$ & $85(70.8)$ & 0.20 \\
\hline
\end{tabular}


Table 2. Alcohol-related and Intervention-related Characteristics by Risk Outcomes at 3 and 12 Months

\begin{tabular}{|c|c|c|c|c|c|c|}
\hline & \multicolumn{3}{|l|}{ At 3 months } & \multicolumn{3}{|l|}{ At 12 months } \\
\hline & $\begin{array}{l}\text { Individuals who } \\
\text { are not at risk } \\
(n=125)\end{array}$ & $\begin{array}{l}\text { Individuals who } \\
\text { remained at risk } \\
(n=122)\end{array}$ & p-value & $\begin{array}{l}\text { Individuals who } \\
\text { are not at risk } \\
(n=102)\end{array}$ & $\begin{array}{l}\text { Individuals who } \\
\text { remained at risk } \\
(n=120)\end{array}$ & p-value \\
\hline $\begin{array}{l}\text { Average number of alcoholic drinks per } \\
\text { week, mean } \pm \mathrm{SD}\end{array}$ & $12.7 \pm 6.2$ & $18 \pm 7.1$ & $<.001$ & $13.4 \pm 7$ & $17 \pm 6.5$ & $<.001$ \\
\hline Daily use of alcohol, n (\%) & $70(56)$ & $98(80.3)$ & $<.001$ & $62(60.8)$ & $93(77.5)$ & 0.007 \\
\hline $\begin{array}{l}\text { Average number of risks at baseline, } \\
\text { mean } \pm \mathrm{SD}\end{array}$ & $2.3 \pm 1.4$ & $3.5 \pm 1.8$ & $<.001$ & $2.3 \pm 1.5$ & $3.4 \pm 1.7$ & $<.001$ \\
\hline $\begin{array}{l}\text { Physician reported providing advice to } \\
\text { reduce drinking, } \mathrm{n}(\%)\end{array}$ & $103(82.4)$ & $107(87.7)$ & 0.24 & $83(81.4)$ & $108(90)$ & 0.06 \\
\hline $\begin{array}{l}\text { Completed at least one health educator } \\
\text { call, } \mathrm{n}(\%)\end{array}$ & 117 (93.6) & $102(83.6)$ & 0.01 & $94(92.2)$ & $105(87.5)$ & 0.26 \\
\hline
\end{tabular}

tion characteristics, and whether a physician documented advice given to reduce drinking between those who completed at least one call and those who did not revealed no significant differences between the two groups (all p-values $\geq 0.10$ ).

\section{Risk Outcomes at 3 and 12 Months}

Two hundred fifty-eight individuals $(83.2 \%$ of intervention subjects) had data on risk scores at either 3 or 12 months, and $212(68.4 \%)$ of these individuals had risk scores for both time periods. Among those with both 3- and 12-month risk score data, $74.5 \%$ of individuals had the same risk outcome at 3 and 12 months (77 remained not at-risk and 81 remained atrisk for both periods).

\section{Sample Sociodemographic and Health-related Characteristics Associated with Risk Outcomes}

As shown in Table 1, there were several differences between those who were not at-risk and those who remained at-risk during the follow-up periods. Univariate analyses showed that those who achieved not at-risk outcome at 3 months were more likely to be women, Hispanic or non-white, and have lower levels of education compared to individuals who remained at-risk. However, these differences were no longer significant at 12 months.

\section{Sample Alcohol-related and Intervention-related Characteristics Associated with Risk Outcomes}

Subjects who were not at-risk at 3 or 12 months consume less alcohol, drink less frequently, and had lower baseline risk scores compared to those who remained at-risk. Those who remained at-risk were more likely to be at risk due to amount of alcohol use, use of alcohol with comorbidities or medications, or binge drinking compared to individuals who achieved not at-risk outcome.

Receiving physicians' advice was not associated with risk outcomes at either 3 or 12 months. Completing at least one health educator call was associated with not at-risk outcome at 3 months but not at 12 months (Table 2).

\section{Effects of Completing Health Educator Calls on Not At-Risk Outcome}

Adjusted odds ratios showed that completing all three health educator calls increased the odds of achieving not at-risk outcome at 3 months by more than five times compared to completing no calls. However, the magnitude of this effect was no longer seen at 12 months. Completing only one or two calls did not significantly increase the odds of achieving not at-risk outcome at either 3 or 12 months compared to completing no calls (Table 3).

\section{DISCUSSION}

At-risk older drinkers who completed all three health educator calls were more likely to transition to not at-risk status at 3 months compared to those who did not complete any calls. However, the effectiveness of the health educator calls was no longer apparent at 12 months. Prior to the health educator call, all subjects had received personalized risk reports, visits with their physicians, and educational booklets on alcohol and aging.

Table 3. Factors Associated with Achieving Not At-Risk Outcome at 3 and 12 Months $^{a}$

\begin{tabular}{|c|c|c|c|c|}
\hline & \multicolumn{2}{|l|}{3 Months } & \multicolumn{2}{|l|}{12 Months } \\
\hline & OR $(95 \% \mathrm{Cl})$ & $p$-value & OR $(95 \% \mathrm{Cl})$ & $\mathrm{p}$-value \\
\hline \multicolumn{5}{|c|}{ Number of health educator calls completed: } \\
\hline One or two & $2.43(0.83-7.11)$ & 0.10 & $1.57(0.52-4.71)$ & 0.42 \\
\hline All three calls & $5.31(1.92-14.7)$ & 0.001 & $2.01(0.71-5.67)$ & 0.18 \\
\hline Number of alcoholic drinks per week & $0.95(0.89-1.01)$ & 0.08 & $0.98(0.92-1.05)$ & 0.60 \\
\hline Daily use of alcohol & $0.52(0.24-1.11)$ & 0.09 & $0.54(0.25-1.19)$ & 0.13 \\
\hline Baseline risk score & $0.73(0.58-0.92)$ & 0.007 & $0.70(0.55-0.88)$ & 0.003 \\
\hline
\end{tabular}

${ }^{a}$ Mixed-effects logistic regression adjusted for age, gender, race/ethnicity, education, ability to do strenuous and heavy work, and physician advice 
It is possible that motivation to change at baseline played a role in both completing health educator calls and eventual reduction in risky drinking at 3 months. If motivation was the key factor, we would have expected individuals who completed one or two calls to fare better than those who did not complete any calls. The findings instead indicated that individuals who completed only one or two calls did not have significant differences in any outcomes at 3 or 12 months compared to those who did not complete any calls. This pattern of results supported a decreased likelihood that motivation was a confounder and suggested that having at least three calls, rather than fewer, was necessary to reduce drinking among older at-risk drinkers.

The telephone has been utilized effectively for monitoring and treatment of various disorders, including depression, ${ }^{37-40}$ tobacco cessation, ${ }^{41,42}$ and alcohol misuse. ${ }^{31,32,38}$ Studies focusing on use of telephone-based intervention in alcohol misuse found reductions in the number of risky drinking days in men with alcohol dependence, ${ }^{31}$ in total alcohol consumption in at-risk adult drinkers in primary care practices, ${ }^{32}$ and in impaired driving among adult patients who screened positive for high-risk alcohol use in the emergency department. ${ }^{33}$ A study that focused on older drinkers in primary and specialty care at the Veterans Administration found that telephone management induced greater reduction in drinking compared to usual care. ${ }^{38}$

There are several advantages of using telephone-based intervention. These interventions can be performed by nonphysician providers, ${ }^{31-33,38,40-42}$ potentially circumventing some of the current barriers that exist in implementing brief intervention in primary care, and increasing the number of health professionals available to reach more at-risk drinkers. Use of the telephone could potentially yield higher rates of selfreported alcohol-related harm compared to face-to-face interview, fostered by the increased anonymity associated with telephone conversations. ${ }^{43}$

In our study, the effect of a health educator call on risk outcomes was evident at 3 months, but the impact was less at 12 months. We found that the majority of subjects' risk outcomes remained the same for both 3 and 12 months, suggesting that most of the changes had occurred early in the trial. Additionally, the lack of effect seen at 12 months could be due to the fact that all three calls were completed during the first eight weeks of the trial, and the effects on at-risk outcomes and drinking could potentially be sustained longer if the calls had been spread out more evenly or occurred more frequently throughout the trial. Other studies of telephonebased intervention included more frequent or intensive telephone counseling sessions, ${ }^{31,32,38,44}$ and one study found beneficial effects up to 24 months in younger adults. ${ }^{44}$ Future studies would be needed to address whether more frequent or intensive telephone sessions would have more impact on long term outcomes for older at-risk drinkers.

There were several limitations of this study. The health educator call was a component of a multi-faceted intervention for older at-risk drinkers in this trial. Therefore, it is possible that other parts of the intervention influenced the impact of the telephone calls. Since health educator calls were embedded in the overall intervention strategy, we were unable to conclude whether use of telephone calls alone would be effective in reducing risky alcohol use among older drinkers. Also, not every intervention subject completed a call, and systemic differences among the two groups, including motivation for change, could potentially bias the result. Although it is not surprising that individuals who did not complete health educator calls had lower rates of completing follow-up surveys, this differential response between those who completed at least a call and those who did not complete any call could potentially introduce bias. We attempted to control for the differential response in the final models by including variables associated with completion of surveys. Furthermore, our sample was composed mainly of non-Hispanic white men, and findings need to be replicated among more diverse group of older adults to improve generalizability of our findings.

In conclusion, we found that health educator telephone call was moderately efficacious in short-term reduction of risky alcohol use among older drinkers in primary care settings. However, the effect did not persist at 12 months, and further research would be needed to determine if telephone-based intervention can be effective in long term reduction of risky drinking among older adults. These findings provide the first data regarding the impact of telephone-based intervention among older adults identified as at-risk drinkers when considering not only the amount they drink but also comorbidities and use of medications that may increase alcohol-related harm. This study adds to the literature suggesting that telephone can be a useful strategy to deliver interventions for unhealthy alcohol use.

Acknowledgments: Funding sources: Special Fellowship in Advanced Geriatrics, VA Greater Los Angeles Healthcare System, and the National Institutes of Health, National Institute on Alcoholism and Alcohol Abuse (AA013937 and AA15957)

Conflict of Interest: None disclosed.

Corresponding Author: James C. Lin, MD, MBA; Special Fellowship in Advanced Geriatrics, VA Greater Los Angeles Healthcare System, 11301 Wilshire Ave, Building 220, Room 302, Los Angeles, CA 90073, USA (e-mail: jlin1207@ucla.edu).

\section{REFERENCES}

1. Hanson GR, Li TK. Public health implications of excessive alcohol consumption. JAMA. 2003;289(8):1031-2.

2. Mehta MM, Moriarty KJ, Proctor D, Bird M, Darling W. Alcohol misuse in older people: heavy consumption and protean presentations. J Epidemiol Community Health. 2006;60(12):1048-52.

3. Moore AA, Morgenstern H, Harawa NT, Fielding JE, Higa J, Beck JC. Are older hazardous and harmful drinkers less likely to participate in health-related behaviors and practices as compared with nonhazardous drinkers? J Am Geriatr Soc. 2001;49(4):421-30

4. Flowers NT, Naimi TS, Brewer RD, Elder RW, Shults RA, Jiles R. Patterns of alcohol consumption and alcohol-impaired driving in the United States. Alcohol Clin Exp Res. 2008;32(4):639-44.

5. Mukamal KJ, Mittleman MA, Longstreth WT Jr, Newman AB, Fried LP, Siscovick DS. Self-reported alcohol consumption and falls in older adults: cross-sectional and longitudinal analyses of the cardiovascular health study. J Am Geriatr Soc. 2004;52(7):1174-9.

6. Saitz R. Clinical practice. Unhealthy alcohol use. N Engl J Med. 2005;352(6):596-607.

7. Adams WL, Cox NS. Epidemiology of problem drinking among elderly people. Int J Addict. 1995;30(13-14):1693-716.

8. Projected Population of the United States, by Age and Sex: 2000 to 2050. http://www.census.gov/population/www/projections/usinterimproj/ natprojtab02a.pdf. Accessed December, 2009. 
9. Merrick EL, Horgan CM, Hodgkin D, et al. Unhealthy drinking patterns in older adults: prevalence and associated characteristics. J Am Geriatr Soc. 2008;56(2):214-23.

10. Moore AA, Morton SC, Beck JC, et al. A new paradigm for alcohol use in older persons. Med Care. 1999;37(2):165-79.

11. Vestal RE, McGuire EA, Tobin JD, Andres R, Norris AH, Mezey E. Aging and ethanol metabolism. Clin Pharmacol Ther. 1977;21(3):34354.

12. Kirchner JE, Zubritsky C, Cody M, et al. Alcohol consumption among older adults in primary care. J Gen Intern Med. 2007;22(1):92-7.

13. Moore AA, Whiteman EJ, Ward KT. Risks of combined alcohol/ medication use in older adults. Am J Geriatr Pharmacother. 2007;5(1): 64-74.

14. Aira M, Hartikainen S, Sulkava R. Community prevalence of alcohol use and concomitant use of medication-a source of possible risk in the elderly aged 75 and older? Int J Geriatr Psychiatry. 2005;20(7):680-5.

15. Pringle KE, Ahern FM, Heller DA, Gold CH, Brown TV. Potential for alcohol and prescription drug interactions in older people. J Am Geriatr Soc. 2005;53(11):1930-6.

16. Moore AA, Giuli L, Gould R, et al. Alcohol use, comorbidity, and mortality. J Am Geriatr Soc. 2006;54(5):757-62.

17. Ockene JK, Adams A, Hurley TG, Wheeler EV, Hebert JR. Brief physician- and nurse practitioner-delivered counseling for high-risk drinkers: does it work? Arch Intern Med. 1999;159(18):2198-205.

18. Reiff-Hekking S, Ockene JK, Hurley TG, Reed GW. Brief physician and nurse practitioner-delivered counseling for high-risk drinking. Results at 12-month follow-up. J Gen Intern Med. 2005;20(1):7-13.

19. Fleming MF, Barry KL, Manwell LB, Johnson K, London R. Brief physician advice for problem alcohol drinkers. A randomized controlled trial in community-based primary care practices. JAMA. 1997;277(13): $1039-45$.

20. Fleming MF, Manwell LB, Barry KL, Adams W, Stauffacher EA. Brief physician advice for alcohol problems in older adults: a randomized community-based trial. J Fam Pract. 1999;48(5):378-84.

21. Kaner EF, Beyer F, Dickinson HO, et al. Effectiveness of brief alcohol interventions in primary care populations. Cochrane Database Syst Rev. 2007:2:CD004148.

22. Fleming MF, Mundt MP, French MT, Manwell LB, Stauffacher EA Barry KL. Brief physician advice for problem drinkers: long-term efficacy and benefit-cost analysis. Alcohol Clin Exp Res. 2002;26(1):36-43.

23. Saitz R, Horton NJ, Cheng DM, Samet JH. Alcohol counseling reflects higher quality of primary care. J Gen Intern Med. 2008;23(9):1482-6.

24. Whitlock EP, Polen MR, Green CA, Orleans T, Klein J. Behavioral counseling interventions in primary care to reduce risky/harmful alcohol use by adults: a summary of the evidence for the U.S. Preventive Services Task Force. Ann Intern Med. 2004;140(7):557-68.

25. Friedmann PD, McCullough D, Chin MH, Saitz R. Screening and intervention for alcohol problems. A national survey of primary care physicians and psychiatrists. J Gen Intern Med. 2000;15(2):84-91.

26. Roche AM, Freeman T. Brief interventions: good in theory but weak in practice. Drug Alcohol Rev. 2004;23(1):11-8.

27. Beich A, Gannik D, Malterud K. Screening and brief intervention for excessive alcohol use: qualitative interview study of the experiences of general practitioners. BMJ. 2002;325(7369):870.
28. Roche AM, Hotham ED, Richmond RL. The general practitioner's role in AOD issues: overcoming individual, professional and systemic barriers. Drug Alcohol Rev. 2002;21(3):223-30.

29. Barry KL, Blow FC, Willenbring ML, McCormick R, Brockmann LM, Visnic S. Use of Alcohol Screening and Brief Interventions $<$ br $/>$ in Primary Care Settings:Implementation and Barriers. Subst Abus. 2004;25(1):27-36.

30. Saitz R, Horton NJ, Sullivan LM, Moskowitz MA, Samet JH. Addressing alcohol problems in primary care: a cluster randomized, controlled trial of a systems intervention. The screening and intervention in primary care (SIP) study. Ann Intern Med. 2003;138(5):372-82.

31. Brown RL, Saunders LA, Bobula JA, Mundt MP, Koch PE. Randomized-controlled trial of a telephone and mail intervention for alcohol use disorders: three-month drinking outcomes. Alcohol Clin Exp Res. 2007;31(8):1372-9

32. Bischof G, Grothues JM, Reinhardt S, Meyer C, John U, Rumpf HJ. Evaluation of a telephone-based stepped care intervention for alcoholrelated disorders: a randomized controlled trial. Drug Alcohol Depend. 2008;93(3):244-51.

33. Mello MJ, Longabaugh R, Baird J, Nirenberg T, Woolard R. DIAL: a telephone brief intervention for high-risk alcohol use with injured emergency department patients. Ann Emerg Med. 2008;51(6):755-64.

34. Moore AA, Beck JC, Babor TF, Hays RD, Reuben DB. Beyond alcoholism: identifying older, at-risk drinkers in primary care. J Stud Alcohol. 2002;63(3):316-24.

35. Miller WR. Motivational interviewing with problem drinkers. Behav Psychother. 1983;11:147-72.

36. The GLIMMIX Procedure, June 2006: SAS Incorporated; 2006.

37. Oslin DW, Ross J, Sayers S, Murphy J, Kane V, Katz IR. Screening, assessment, and management of depression in VA primary care clinics. The Behavioral Health Laboratory. J Gen Intern Med. 2006;2 1(1):46-50.

38. Oslin DW, Sayers S, Ross J, et al. Disease management for depression and at-risk drinking via telephone in an older population of veterans. Psychosom Med. 2003;65(6):931-7.

39. Simon GE, VonKorff M, Rutter C, Wagner E. Randomised trial of monitoring, feedback, and management of care by telephone to improve treatment of depression in primary care. BMJ. 2000;320(7234):550-4

40. Simon GE, Ludman EJ, Tutty S, Operskalski B, Von Korff M. Telephone psychotherapy and telephone care management for primary care patients starting antidepressant treatment: a randomized controlled trial. JAMA. 2004;292(8):935-42.

41. Curry SJ, McBride C, Grothaus LC, Louie D, Wagner EH. A randomized trial of self-help materials, personalized feedback, and telephone counseling with nonvolunteer smokers. J Consult Clin Psychol. 1995; 63(6): 1005-14.

42. Lando HA, Hellerstedt WL, Pirie PL, McGovern PG. Brief supportive telephone outreach as a recruitment and intervention strategy for smoking cessation. Am J Public Health. 1992;82(1):41-6.

43. Midanik LT, Greenfield TK, Rogers JD. Reports of alcohol-related harm: telephone versus face-to-face interviews. J Stud Alcohol. 2001;62 (1):74-8.

44. McKay JR, Lynch KG, Shepard DS, Pettinati HM. The effectiveness of telephone-based continuing care for alcohol and cocaine dependence: 24-month outcomes. Arch Gen Psychiatry. 2005;62(2):199-207. 